\title{
Association between obesity and magnetic resonance imaging defined patellar tendinopathy in community-based adults: a cross-sectional study
}

Jessica Fairley ${ }^{1}$, Jason Toppi ${ }^{1}$, Flavia M Cicuttini ${ }^{1}$, Anita E Wluka ${ }^{1}$, Graham G Giles ${ }^{1,2,3}$, Jill Cook ${ }^{4}$, Richard O'Sullivan ${ }^{5,6}$ and Yuanyuan Wang ${ }^{1 *}$

\begin{abstract}
Background: Patellar tendinopathy is a common cause of activity-related anterior knee pain. Evidence is conflicting as to whether obesity is a risk factor for this condition. The aim of this study was to determine the relationship between obesity and prevalence of magnetic resonance imaging (MRI) defined patellar tendinopathy in community-based adults.

Methods: 297 participants aged 50-79 years with no history of knee pain or injury were recruited from an existing community-based cohort. Measures of obesity included measured weight and body mass index (BMI), self-reported weight at age of 18-21 years and heaviest lifetime weight. Fat-free mass and fat mass were measured using bioelectrical impedance. Participants underwent MRI of the dominant knee. Patellar tendinopathy was defined on both T1- and T2-weighted images.

Results: The prevalence of MRI defined patellar tendinopathy was 28.3\%. Current weight (OR per $\mathrm{kg}=1.04,95 \%$ $\mathrm{Cl} 1.01-1.06, \mathrm{P}=0.002), \mathrm{BMI}\left(\mathrm{OR}\right.$ per $\left.\mathrm{kg} / \mathrm{m}^{2}=1.10,95 \% \mathrm{Cl} 1.04-1.17, \mathrm{P}=0.002\right)$, heaviest lifetime weight (OR per $\mathrm{kg}=1.03$, $95 \% \mathrm{Cl} 1.01-1.05, \mathrm{P}=0.007$ ) and weight at age of $18-21$ years (OR per $\mathrm{kg}=1.03,95 \% \mathrm{Cl} 1.00-1.07, \mathrm{P}=0.05$ ) were all positively associated with the prevalence of patellar tendinopathy. Neither fat mass nor fat-free mass was associated with patellar tendinopathy.

Conclusion: MRI defined patellar tendinopathy is common in community-based adults and is associated with current and past history of obesity assessed by BMI or body weight, but not fat mass. The findings suggest a mechanical pathogenesis of patellar tendinopathy and patellar tendinopathy may be one mechanism for obesity related anterior knee pain.
\end{abstract}

Keywords: Obesity, Patellar tendinopathy, Body mass index, Weight, Fat mass, Magnetic resonance imaging

\section{Background}

Patellar tendinopathy is a clinical condition causing activity-related anterior knee pain and subsequent dysfunction $[1,2]$. It has been most commonly described in athletes participating in sports that involve jumping, earning it the name "jumper's knee", where the tendon matrix is affected by repetitive stress on the tendon

\footnotetext{
*Correspondence: yuanyuan.wang@monash.edu

'School of Public Health and Preventive Medicine, Monash University, Alfred Hospital, Melbourne VIC 3004, Australia

Full list of author information is available at the end of the article
}

$[3,4]$, but patellar tendinopathy has also been found in people who do not participate in jumping sports $[3,5]$. The prime pathological feature of patellar tendinopathy histologically is characterised by mucoid degeneration which can be detected using ultrasonography or magnetic resonance imaging (MRI) [6-8]. Although not all individuals with evidence of patellar tendinopathy on imaging have symptoms [6,9], those tendon abnormalities identified by imaging modality in asymptomatic individuals predict the development of tendon-related symptoms and disability $[10,11]$. In addition, these imaging changes are not always 
present in those with symptomatic patellar tendinopathy. This may in part be due to the incorrect attribution of symptoms to patellar tendinopathy when other joint pathology may be the source of symptoms [12,13]. MRI has advanced our understanding of factors that contribute to pain and function in the tibiofemoral joint $[14,15]$. These structural changes in and around the knee joint develop on a continuum from the healthy joint to the diseased joint.

Pathology in the patellofemoral compartment is increasingly recognised as a source of pain and impaired function with aging $[16,17]$. However, there has been relatively little work at the patellofemoral joint, compared with the tibiofemoral joint, examining structures such as tendons. Most of the instruments examining pain and function in epidemiological and clinical studies of older people focus on the tibiofemoral compartment and do not address symptoms that may be due to patellar tendinopathy, such as activity-related anterior knee pain through important functional activities such as knee bending.

A large body of work on patellar tendinopathy performed using athletic populations has identified male gender [18-20] and body mass $[3,21,22]$ as risk factors. The evidence is conflicting about any association between patellar tendinopathy and obesity, a condition which is known to increase strain on joints and tendons throughout the body, especially the weight-bearing joints, with some studies showing no association [18,23]. Any association between body composition and risk of patellar tendinopathy is also inconclusive $[21,23]$. In older populations, obesity has been related to knee pain and poor function $[24,25]$. It is possible that patellar tendon pathology may be one of the mechanisms for obesity related anterior knee pain in older people.

Thus the aim of the current study was to determine the prevalence of MRI defined patellar tendinopathy in community-based asymptomatic adults and whether it is associated with obesity and body composition. Examining this in an asymptomatic population may reduce the potential for any associations identified being due to reverse causation. We hypothesised that obesity would be associated with increased risk of MRI defined patellar tendinopathy in community-based individuals.

\section{Methods}

\section{Study participants}

The study was conducted within the Melbourne Collaborative Cohort Study (MCCS), a prospective cohort study of 41,514 residents of Melbourne, Australia. The aim of the MCCS is to examine the role of lifestyle and genetic factors in the risk of cancer and chronic diseases [26]. Participants for the current study were recruited from within the MCCS between 2003-2004 as described [27]. Briefly, participants were eligible if they were aged between 50-79 years without any of the following exclusion criteria: a clinical diagnosis of knee osteoarthritis as defined by American College of Rheumatology criteria [28]; knee pain lasting for $>24$ hours in the last 5 years; a previous knee injury requiring non-weight bearing treatment for $>24$ hours or surgery (including arthroscopy); or a history of any form of arthritis diagnosed by a medical practitioner. A further exclusion criterion was a contraindication to MRI including pacemaker, metal sutures, presence of shrapnel or iron filings in the eye, or claustrophobia. The study was approved by The Cancer Council Victoria's Human Research Ethics Committee and Monash University Human Research Ethics Committee. All participants gave written informed consent.

\section{Anthropometric measurements}

At baseline (2003-2004), height (cm) was measured using a stadiometer with shoes removed, and weight $(\mathrm{kg})$ was measured with bulky clothing removed. Body mass index (BMI) was calculated from these data [weight $(\mathrm{kg}) /$ height $^{2}$ $\left(\mathrm{m}^{2}\right)$ ]. Bioelectrical impedance analysis was performed with a single frequency $(50 \mathrm{kHz})$ electric current produced by a BIA-101A RJL system analyser (RJL systems, Detroit, $\mathrm{MI})$. Resistance and reactance were measured with participants in a supine position. Non-adipose mass, hereafter termed fat-free mass, was estimated as $9.1536+(0.4273 \times$ height ${ }^{2} /$ resistance $)+(0.1926 \times$ weight $)+(0.0667 \times$ reactance $)$ for males, and $7.7435+\left(0.4542 \times\right.$ height $^{2} /$ resistance $)+^{-}$ $(0.119 \times$ weight $)+(0.0455 \times$ reactance $)$ for females [29] Adipose mass, hereafter termed fat mass, was subsequently calculated as weight - fat-free mass. There is evidence for the validity and reliability of bioelectrical impedance analysis in determining body composition [30,31]. A particular issue for bioelectric impedance analysis is the absence of a standard equation to estimate fat-free mass. We chose a formula developed using subjects of similar ethnicity, age, and BMI distribution to the MCCS population [29] and validated using sound statistical techniques. At MCCS baseline (1990-1994), participants were also asked to recall their weight when they were aged between 18-21 years and their heaviest lifetime weight.

\section{MRI acquisition}

An MRI of the dominant knee (defined as the lower-limb from which the participant stepped off from when initiating gait) was performed between October 2003 and December 2004 and repeated approximately 2 years later. Knees were imaged in the sagittal plane on a $1.5-\mathrm{T}$ whole body magnetic resonance unit (Gyroscan Intera, Philips Medical Systems, Eindhoven, The Netherlands) using a commercial transmit-receive extremity coil. The following sequence and parameters were used: a $\mathrm{T}_{1}$ weighted fat suppressed 3D gradient recall acquisition in the steady state; flip angle 55 degrees; repetition time 
$58 \mathrm{msec}$; echo time $12 \mathrm{msec}$; field of view $16 \mathrm{~cm}$; 60 partitions; $512 \times 512$ matrix; one acquisition time $11 \mathrm{~min}$ 56 sec. Sagittal images were obtained at a partition thickness of $1.5 \mathrm{~mm}$ and an in-plane resolution of $0.31 \times 0.31 \mathrm{~mm}(512 \times 512$ pixels $)$. In addition, a coronal $\mathrm{T}_{2}$-weighted fat-saturated acquisition, repetition time 3500-3800 msec, echo time $50 \mathrm{msec}$, with a slice thickness of $3.0 \mathrm{~mm}$, a $1.0 \mathrm{~mm}$ interslice gap, 1 excitation, a field of view of $13 \mathrm{~cm}$, and a matrix of $256 \times 192$ pixels was also obtained.

\section{Assessment of patellar tendinopathy}

Patellar tendinopathy was defined as an area of increased signal intensity of characteristic pattern, size and distribution on at least two adjacent slices in the proximal region of the inferior patellar tendon. Two trained observers, who were blinded to participant characteristics, assessed the presence of lesions for each participant in T1-weighted fat-saturated sagittal images. T2-weighted fat-saturated coronal images were used to confirm the presence of patellar tendinopathy and ensure a magic angle effect was not contributing to positive results. When echo time is short, increased signal intensity can be observed in the absence of pathology, which is known as the magic angle effect. To overcome the magic angle effect a T2-weighted sequence can be applied [32], which was done in this study. The patellar tendon was graded as either 'definite tendinopathy' or 'no tendinopathy' [9,32,33] (Figure 1). The reproducibility for determination of definite patellar tendinopathy was assessed using 50 randomly selected knee MRIs (intraclass coefficient correlation was 0.94).

\section{Statistical analysis}

The outcome measure was the prevalence of MRI defined patellar tendinopathy. The exposures of interest were different body mass measurements (weight, BMI, and body composition). Binary logistic regression was used to examine the association between body mass measures and the prevalence of MRI defined patellar tendinopathy, adjusting for confounders of age, gender, and height (for body weight only). A p-value of less than 0.05 (two-tailed) was regarded as statistically significant. All analyses were performed using IBM SPSS version 20.

\section{Results}

The characteristics of the 297 participants aged between 50 and 79 years (mean 58.0 years, SD 5.5 years) are presented in Table 1. Participants with patellar tendinopathy ( $\mathrm{n}=84,28.3 \%)$ were more likely to be men $(\mathrm{P}<0.001)$, taller $(\mathrm{P}=0.02)$, and had greater body weight $(\mathrm{P}<0.001)$ and BMI ( $\mathrm{P}=0.001)$ than those without patellar tendinopathy. Weight at the age of 18-21 years and heaviest lifetime weight were also greater for those with tendinopathy compared with those without (all $\mathrm{P}<0.001$ ). Two hundred and thirty three $(78.5 \%)$ participants had current fat mass and fat free mass measures. Participants with patellar tendinopathy had higher fat free mass than those without $(\mathrm{P}<0.001)$, but there was no significant difference in fat mass between the two groups $(\mathrm{P}=0.21)$.

\section{Relationship between weight and BMI and prevalence of patellar tendinopathy}

In univariate analyses, BMI, body weight, weight at ages of 18-21 years and heaviest lifetime weight were all positively associated with the prevalence of patellar tendinopathy (Table 2). After adjusting for age and gender, BMI remained positively associated with the prevalence of patellar tendinopathy (odds ratio (OR) 1.10, 95\% confidence interval (CI) 1.04 - 1.17). Body weight (OR 1.04, 95\% CI 1.01 - 1.06) and heaviest lifetime weight (OR 1.03, 95\% CI 1.01 - 1.05) also remained positively associated with the prevalence of patellar tendinopathy after adjusting for age, gender and height. Weight at ages of 18-21 years was marginally associated with the prevalence of patellar tendinopathy (OR 1.03, 95\% CI 1.00 - 1.07) (Table 2).

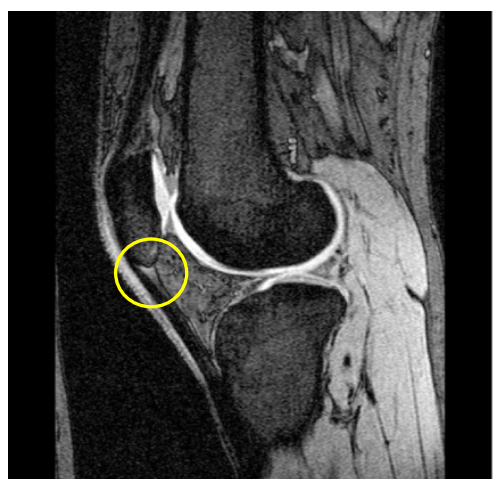

A

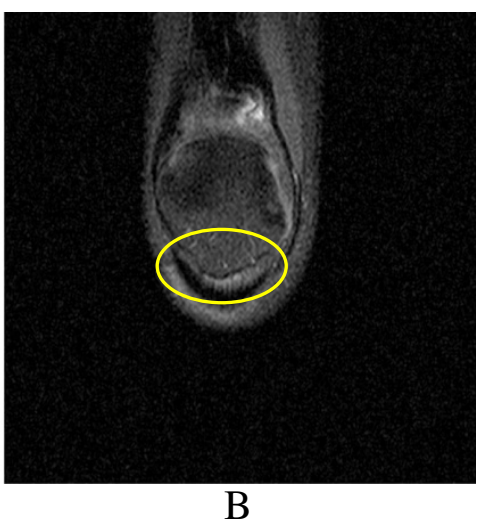

B

Figure 1 Patellar tendinopathy on MRI on A. T1-weighted fat-saturated sagittal MRl; and $\mathbf{B}$. T2-weighted fat-saturated coronal MRI. 
Table 1 Characteristics of study population

\begin{tabular}{lccc}
\hline & $\begin{array}{c}\text { No tendinopathy } \\
(\mathbf{n}=\mathbf{2 1 3})\end{array}$ & $\begin{array}{c}\text { Tendinopathy } \\
(\mathbf{n}=\mathbf{8 4})\end{array}$ & P value* $^{*}$ \\
\hline Age (years) & $57.6(5.2)$ & $59.0(6.0)$ & 0.06 \\
Female, number (\%) & $147(69)$ & $39(46)$ & $<0.001$ \\
Height (cm) & $167.3(8.7)$ & $170.0(9.5)$ & 0.02 \\
Weight (kg) & $71.3(13.8)$ & $78.9(14.2)$ & $<0.001$ \\
Body mass index (kg/m²) & $25.4(4.2)$ & $27.2(4.2)$ & 0.001 \\
Fat-free mass $(\mathrm{kg})^{\dagger}$ & $44.4(9.3)$ & $51.7(10.7)$ & $<0.001$ \\
Fat mass (kg) & $26.1(9.0)$ & $27.8(8.4)$ & 0.21 \\
Weight at ages of 18-21 & $59.2(11.1)$ & $64.3(11.3)$ & $<0.001$ \\
years (kg) & & & \\
Heaviest lifetime & $71.1(14.1)$ & $78.7(13.7)$ & $<0.001$ \\
weight (kg) & & &
\end{tabular}

Values are reported as mean (SD) except for percentages.

*Differences between those with and without patellar tendinopathy, using independent samples $t$ test or Chi-squared test.

${ }^{\dagger}$ Data available on 233 participants.

\section{Relationship between body composition and prevalence of patellar tendinopathy}

In univariate analyses, fat free mass was positively associated with the prevalence of patellar tendinopathy but there was no significant association with fat mass (Table 3). After adjusting for age and gender, the significant association with fat free mass remained (OR 1.08, 95\% CI 1.01 - 1.15) while fat mass was weakly associated with the prevalence of patellar tendinopathy (OR 1.03, 95\% CI 1.00 - 1.07). After adjustment for fat free mass and fat mass along with age and gender, neither fat free mass (OR 1.07, 95\% CI 0.99 - 1.16) nor fat mass (OR 1.01, 95\% CI 0.96 - 1.05) were significantly associated with the prevalence of patellar tendinopathy (Table 3).

\section{Discussion}

In community-based adults without clinical knee disease, MRI defined patellar tendinopathy was common (28.3\%). There was an increased risk of MRI defined patellar tendinopathy associated with both current and past history of obesity as assessed by either BMI or body weight. Neither fat mass nor fat free mass was associated with the risk of MRI defined patellar tendinopathy when they were co-adjusted.
Existing studies aimed at determining the prevalence of patellar tendinopathy have used young athletic populations, both elite and non-elite. Patellar tendinopathy, which is also known as "jumper's knee", is traditionally considered to be mainly a disease of athletes, especially those participating in sports involving significant force through the patellar tendon [2]. The prevalence and significance of patellar tendinopathy have been illustrated in a number of samples of athletes from different sports, with prevalence ranging from $2.5 \%$ to $41 \%$ [3,20,34]. Data are limited regarding the prevalence of patellar tendinopathy in non-athletic, healthy middle-aged to older populations. Our study demonstrated that the prevalence of MRI defined patellar tendinopathy was $28.3 \%$ in a communitybased population of adults without clinical knee disease, supporting the findings from a previous study that prevalence of patellar tendinopathy is increased in older populations [35].

There is conflicting evidence for an association between obesity and patellar tendinopathy. A clinical diagnosis of patellar tendinopathy has been reported to be associated with increased weight $[3,21,22]$ and BMI $[21,22]$ by a number of studies of athletes, but there is also evidence suggesting that weight is not a significant risk factor for patellar tendinopathy $[18,23]$. Our study has demonstrated a clear relationship between current and past history of obesity and MRI defined patellar tendinopathy in an asymptomatic older population. The value of examining a population without clinical symptoms is important since it is unlikely that the knee pain and function limitation cause reduced mobility and increased obesity, i.e. the potential reverse causality. It is more likely that obesity contributes to these pathological tendon abnormalities. Thus we highlight the unique contribution of our results from an unselected middle-aged population in contributing to knowledge of patellar tendinopathy.

We found no relationship between body composition (fat mass and fat free mass) and MRI defined patellar tendinopathy when adjusting for age, gender, fat mass and fat free mass. This is supported by the findings from a previous study that there is no clear link between body composition and patellar tendinopathy in elite female basketball players [23]. On the other hand, another study

Table 2 Associations of weight and body mass index with prevalence of patellar tendinopathy

\begin{tabular}{|c|c|c|c|c|}
\hline & \multicolumn{2}{|c|}{ Univariate analysis } & \multicolumn{2}{|c|}{ Multivariate analysis } \\
\hline & Odds ratio $(95 \% \mathrm{Cl})$ & $P$ value & Odds ratio $(95 \% \mathrm{Cl})$ & $P$ value \\
\hline Body mass index $\left(\mathrm{kg} / \mathrm{m}^{2}\right)^{1}$ & $1.10(1.04,1.17)$ & 0.001 & $1.10(1.04,1.17)$ & 0.002 \\
\hline Weight $(\mathrm{kg})^{2}$ & $1.04(1.02,1.06)$ & $<0.001$ & $1.04(1.01,1.06)$ & 0.002 \\
\hline Weight at ages of 18-21 years $(\mathrm{kg})^{2}$ & $1.04(1.02,1.07)$ & 0.001 & $1.03(1.00,1.07)$ & 0.05 \\
\hline Heaviest lifetime weight $(\mathrm{kg})^{2}$ & $1.04(1.02,1.06)$ & $<0.001$ & $1.03(1.01,1.05)$ & 0.007 \\
\hline
\end{tabular}

adjusting for age and gender.

${ }^{2}$ adjusting for age, gender, and height. 
Table 3 Associations between body composition and prevalence of patellar tendinopathy

\begin{tabular}{|c|c|c|c|c|c|c|}
\hline & \multicolumn{2}{|c|}{ Univariate analysis } & \multicolumn{2}{|c|}{ Multivariate analysis $^{1}$} & \multicolumn{2}{|c|}{ Multivariate analysis ${ }^{2}$} \\
\hline & Odds ratio $(95 \% \mathrm{Cl})$ & $P$ value & Odds ratio $(95 \% \mathrm{Cl})$ & $P$ value & Odds ratio $(95 \% \mathrm{Cl})$ & $P$ value \\
\hline Fat free mass $(\mathrm{kg})$ & $1.07(1.04,1.10)$ & $<0.001$ & $1.08(1.01,1.15)$ & 0.02 & $1.07(0.99,1.16)$ & 0.10 \\
\hline Fat mass (kg) & $1.02(0.99,1.05)$ & 0.21 & $1.03(1.00,1.07)$ & 0.08 & $1.01(0.96,1.05)$ & 0.78 \\
\hline
\end{tabular}

1adjusting for age and gender.

${ }^{2}$ adjusting for age, gender, fat free mass and fat mass.

of competitive volleyball players reported an association between central obesity assessed by waist circumference and the risk of patellar tendinopathy [21]. The reasons for the discrepancy of findings are not known, but may be due to different study populations (different age group, gender, and athletes vs. non-athletes) and different methods used to assess body composition and patellar tendinopathy. Further investigations are needed.

Our findings of a positive association between obesity (past and present) and MRI defined patellar tendinopathy and no association with body composition suggest a mechanical effect of obesity on patellar tendinopathy. This mechanism is supported by existing literature that patellar tendinopathy is a disease of athletes who have repeated heavy loading through the patellar tendon, especially sports involving jumping. Differences in prevalence have been established for different sporting populations $[3,20,34]$; the interpretation being that the degree of tendon strain varies between different sports. Previous studies have shown a direct relationship between the number of training sessions and physically demanding work and the risk of patellar tendinopathy [23,36,37]. It is thus of interest that increased mechanical load through the knee due to obesity as assessed by either BMI or body weight is significantly associated with the risk of patellar tendinopathy. This is consistent with the histological basis for patellar tendinopathy which shows matrix changes without any inflammatory component [6,35].

The patellofemoral compartment is being increasingly recognised as a major source of pain and disease $[16,17]$. Patellar tendinopathy can be debilitating and cause significant morbidity [38]. Symptomatic patellar tendinopathy may have a significant negative impact on activity, quality of life and ultimately mental health [4,39]. Although a standardised patellar tendon outcome measure has been developed and extensively validated (Victorian Institute of Sport Assessment, VISA) [40], most traditional and validated instruments for assessing knee pain (such as the Western Ontario and McMaster Universities Osteoarthritis Index (WOMAC) scoring system [41]) focus on the tibiofemoral compartment rather than the patellofemoral compartment. It is well recognized that functional limitations in activities such as knee bending, which have significant impact on knee function, are common in older people. Pathology in the patellofemoral compartment has a significant impact on pain and quality of life $[16,17]$.
Whether symptoms in the region are due to joint pathology or other structures such as patellar tendinopathy has not been examined. Our study suggests that MRI defined patellar tendinopathy is common in older people. Using a non-specific tool (WOMAC), we found some trends for worse knee functions in older people with MRI defined patellar tendinopathy compared with those without (data not shown). However, further work using specific tools will be needed to determine whether patellar tendinopathy explains some of the anterior knee pain and common functional abnormalities seen in older people but not explored by commonly used knee instruments.

This study has limitations. Although the cross-sectional design enabled us to generate hypotheses and add to current evidence using a reasonably sized population, the findings of this cross-sectional study need to be confirmed by longitudinal studies. Further investigation will be required to detail the natural history and other contributing factors for patellar tendinopathy, particularly clarifying whether the disease process has been there since early adulthood or whether it has developed later in life. The participants were asked to recall their weight at 18-21 years at the MCCS inception (1990-1994) when they were aged 40-69 years. Self-report of past weight over a similar period to our study has shown moderate to strong correlation with measured weight (correlation coefficients range from 0.64 to 0.95 ), and these correlations are modified by sex and current weight [42-44]. Moreover, the reliability of self-reported past weight is generally supported in epidemiologic studies [42-44]. The main strength of the study is that patellar tendinopathy has been measured using a non-invasive MRI-based method from both T1- and T2-weighted images to encounter the magic angle effect which showed high reproducibility. In this study we recruited participants with no history of knee disease from a community-based cohort. However we did not specifically ask about knee pain on bending, which would be more specific for patellar tendinopathy. Given the participants did not have significant knee pain, it is unlikely that knee pain restricted activity sufficiently to contribute to the obesity.

\section{Conclusions}

Our study highlights a significant prevalence of MRI defined patellar tendinopathy of $28.3 \%$ in a sample of community-based adults. The positive association of 
MRI defined patellar tendinopathy with both current and past obesity, but not body composition, suggests a mechanical mechanism. The contribution of MRI defined patellar tendinopathy on knee symptoms associated with obesity in the older people warrants further investigation.

\section{Competing interests}

The authors declare that there are no competing interests.

\section{Authors' contributions}

JF was involved in data collection, performed data analysis and interpretation, and drafted the manuscript. JT was involved in data collection and interpretation. FMC was involved in conception and design of the study and data interpretation. AEW was involved in data interpretation. GGG was involved in data collection. JC was involved in data interpretation. RO oversaw imaging and was involved in data collection. YW was involved in conception and design of the study, data analysis and interpretation, and coordinated all suggestions and edits. All authors participated in reviewing and editing the manuscript, and approved the final manuscript.

\section{Acknowledgements}

The Melbourne Collaborative Cohort Study recruitment was funded by VicHealth and The Cancer Council of Victoria. This study was funded by a program grant from the National Health and Medical Research Council (NHMRC; 209057) and was further supported by infrastructure provided by Cancer Council of Victoria. We would like to acknowledge the NHMRC (Project Grant 334150) and Colonial Foundation. We would especially like to thank the study participants who made this study possible.

\section{Author details}

${ }^{1}$ School of Public Health and Preventive Medicine, Monash University, Alfred Hospital, Melbourne VIC 3004, Australia. ${ }^{2}$ Centre for Epidemiology and Biostatistics, Melbourne School of Population and Global Health, The University of Melbourne, Carlton VIC 3053, Australia. ${ }^{3}$ Cancer Epidemiology Centre, Cancer Council Victoria, Carlton VIC 3053, Australia. ${ }^{4}$ Department of Physiotherapy, School of Primary Health Care, Faculty of Medicine, Nursing and Health Sciences, Monash University, Frankston VIC 3199, Australia. ${ }^{5}$ Healthcare Imaging Services, MRI Department, Epworth Hospital, Richmond VIC 3131, Australia. ${ }^{6}$ Department of Medicine, Central Clinical School, Monash University, Melbourne, Australia.

Received: 6 November 2013 Accepted: 25 July 2014

Published: 7 August 2014

\section{References}

1. Khan KM, Cook JL, Kannus P, Maffulli N, Bonar SF: Time to abandon the "tendinitis" myth. BMG 2002, 324(7338):626-627.

2. Visnes $H$, Aandahl HA, Bahr R: Jumper's knee paradox-jumping ability is a risk factor for developing jumper's knee: a 5-year prospective study. $\mathrm{Br} J$ Sports Med 2013, 47(8):503-507.

3. Lian OB, Engebretsen L, Bahr R: Prevalence of jumper's knee among elite athletes from different sports: a cross-sectional study. Am J Sports Med 2005, 33(4):561-567.

4. Kettunen JA, Kvist M, Alanen E, Kujala UM: Long-term prognosis for jumper's knee in male athletes. A prospective follow-up study. Am J Sports Med 2002, 30(5):689-692.

5. Cook JL, Khan KM, Harcourt PR, Grant M, Young DA, Bonar SF: A cross sectional study of 100 athletes with jumper's knee managed conservatively and surgically. The Victorian Institute of Sport Tendon Study Group. Br J Sports Med 1997, 31(4):332-336.

6. Khan KM, Bonar F, Desmond PM, Cook JL, Young DA, Visentini PJ, Fehrmann MW, Kiss ZS, O'Brien PA, Harcourt PR, Dowling RJ, O'Sullivan RM Crichton KJ, Tress BM, Wark JD: Patellar tendinosis (jumper's knee): findings at histopathologic examination, US, and MR imaging. Victorian Institute of Sport Tendon Study Group. Radiology 1996, 200(3):821-827.

7. Popp JE, Yu JS, Kaeding CC: Recalcitrant patellar tendinitis. Magnetic resonance imaging, histologic evaluation, and surgical treatment. Am J Sports Med 1997, 25(2):218-222.
8. Yu JS, Popp JE, Kaeding CC, Lucas J: Correlation of MR imaging and pathologic findings in athletes undergoing surgery for chronic patellar tendinitis. AJR Am J Roentgenol 1995, 165(1):115-118.

9. Reiff DB, Heenan SD, Heron CW: MRI appearances of the asymptomatic patellar tendon on gradient echo imaging. Skeletal Radiol 1995, 24(2):123-126.

10. Cook JL, Khan KM, Kiss ZS, Purdam CR, Griffiths L: Prospective imaging study of asymptomatic patellar tendinopathy in elite junior basketball players. J Ultrasound Med 2000, 19(7):473-479.

11. Comin J, Cook JL, Malliaras P, McCormack M, Calleja M, Clarke A, Connell D: The prevalence and clinical significance of sonographic tendon abnormalities in asymptomatic ballet dancers: a 24-month longitudinal study. Br J Sports Med 2013, 47(2):89-92.

12. El-Khoury GY, Wira RL, Berbaum KS, Pope TL Jr, Monu JU: MR imaging of patellar tendinitis. Radiology 1992, 184(3):849-854.

13. Warden SJ, Kiss ZS, Malara FA, Ooi AB, Cook JL, Crossley KM: Comparative accuracy of magnetic resonance imaging and ultrasonography in confirming clinically diagnosed patellar tendinopathy. Am J Sports Med 2007, 35(3):427-436.

14. Wluka AE, Wolfe R, Stuckey S, Cicuttini FM: How does tibial cartilage volume relate to symptoms in subjects with knee osteoarthritis? Ann Rheum Dis 2004, 63(3):264-268.

15. Felson DT, Chaisson CE, Hill CL, Totterman SM, Gale ME, Skinner KM, Kazis L, Gale DR: The association of bone marrow lesions with pain in knee osteoarthritis. Ann Intern Med 2001, 134(7):541-549.

16. Davies AP, Vince AS, Shepstone L, Donell ST, Glasgow MM: The radiologic prevalence of patellofemoral osteoarthritis. Clin Orthop Relat Res 2002, 402:206-212.

17. Ledingham J, Regan M, Jones A, Doherty M: Radiographic patterns and associations of osteoarthritis of the knee in patients referred to hospital. Ann Rheum Dis 1993, 52(7):520-526.

18. Witvrouw E, Bellemans J, Lysens R, Danneels L, Cambier D: Intrinsic risk factors for the development of patellar tendinitis in an athletic population. A two-year prospective study. Am J Sports Med 2001, 29(2):190-195.

19. Cook JL, Khan KM, Harcourt PR, Kiss ZS, Fehrmann MW, Griffiths L, Wark JD: Patellar tendon ultrasonography in asymptomatic active athletes reveals hypoechoic regions: a study of 320 tendons. Victorian Institute of Sport Tendon Study Group. Clin J Sport Med 1998, 8(2):73-77.

20. Zwerver J, Bredeweg SW, van den Akker-Scheek I: Prevalence of Jumper's knee among nonelite athletes from different sports: a cross-sectional survey. Am J Sports Med 2011, 39(9):1984-1988.

21. Malliaras $\mathrm{P}$, Cook JL, Kent PM: Anthropometric risk factors for patellar tendon injury among volleyball players. Br J Sports Med 2007, 41(4):259-263. discussion 263.

22. Crossley KM, Thancanamootoo K, Metcalf BR, Cook JL, Purdam CR, Warden $\mathrm{SJ}$ : Clinical features of patellar tendinopathy and their implications for rehabilitation. J Orthop Res 2007, 25(9):1164-1175.

23. Gaida JE, Cook JL, Bass SL, Austen S, Kiss ZS: Are unilateral and bilateral patellar tendinopathy distinguished by differences in anthropometry, body composition, or muscle strength in elite female basketball players? Br J Sports Med 2004, 38(5):581-585.

24. Jinks C, Jordan KP, Blagojevic M, Croft P: Predictors of onset and progression of knee pain in adults living in the community. A prospective study. Rheumatology (Oxford) 2008, 47(3):368-374.

25. Mallen CD, Peat G, Thomas E, Lacey R, Croft P: Predicting poor functional outcome in community-dwelling older adults with knee pain: prognostic value of generic indicators. Ann Rheum Dis 2007, 66(11):1456-1461.

26. Giles GG, English DR: The Melbourne collaborative cohort study. IARC SCi Publ 2002, 156:69-70.

27. Baranyay FJ, Wang Y, Wluka AE, English DR, Giles GG, Sullivan RO, Cicuttini FM: Association of bone marrow lesions with knee structures and risk factors for bone marrow lesions in the knees of clinically healthy, community-based adults. Semin Arthritis Rheum 2007, 37(2):112-118.

28. Altman R, Asch E, Bloch D, Bole G, Borenstein D, Brandt K, Christy W, Cooke TD, Greenwald R, Hochberg M, Howell D, Kaplan D, Koopman W, Longley S III, Mankin H, McShane DJ, Medsger T Jr, Meenan R, Mikkelsen W, Moskowitz R, Murphy W, Rothschild B, Segal M, Sokoloff L, Wolfe F: Development of criteria for the classification and reporting of osteoarthritis. Classification of osteoarthritis of the knee. diagnostic and therapeutic criteria committee of the American Rheumatism Association. Arthritis Rheum 1986, 29(8):1039-1049. 
29. Roubenoff R, Baumgartner RN, Harris TB, Dallal GE, Hannan MT, Economos CD, Stauber PM, Wilson PW, Kiel DP: Application of bioelectrical impedance analysis to elderly populations. J Gerontol A Biol Sci Med Sci 1997, 52(3):M129-M136.

30. Kyle UG, Bosaeus I, De Lorenzo AD, Deurenberg P, Elia M, Gomez JM, Heitmann BL, Kent-Smith L, Melchior JC, Pirlich M, Scharfetter H, Schols AM, Pichard C: Bioelectrical impedance analysis-part I: review of principles and methods. Clin Nutr 2004, 23(5):1226-1243.

31. Jackson AS, Pollock ML, Graves JE, Mahar MT: Reliability and validity of bioelectrical impedance in determining body composition. J Appl Physiol 1988, 64(2):529-534.

32. Karantanas AH, Zibis AH, Papanikolaou N: Increased signal intensity on fat-suppressed three-dimensional T1-weighted pulse sequences in patellar tendon: magic angle effect? Skeletal Radiol 2001, 30(2):67-71.

33. McLoughlin RF, Raber EL, Vellet AD, Wiley JP, Bray RC: Patellar tendinitis: MR imaging features, with suggested pathogenesis and proposed classification. Radiology 1995, 197(3):843-848.

34. Lian O, Holen KJ, Engebretsen L, Bahr R: Relationship between symptoms of jumper's knee and the ultrasound characteristics of the patellar tendon among high level male volleyball players. Scand J Med Sci Sports 1996, 6(5):291-296.

35. Warden SJ, Brukner P: Patellar tendinopathy. Clin Sports Med 2003, 22(4):743-759.

36. Ferretti A: Epidemiology of jumper's knee. Sports Med 1986, 3(4):289-295.

37. van der Worp H, Zwerver J, Kuijer PP, Frings-Dresen MH, van den Akker-Scheek I: The impact of physically demanding work of basketball and volleyball players on the risk for patellar tendinopathy and on work limitations. J Back Musculoskelet Rehabil 2011, 24(1):49-55.

38. Lian O, Refsnes PE, Engebretsen L, Bahr R: Performance characteristics of volleyball players with patellar tendinopathy. Am J Sports Med 2003, 31(3):408-413

39. Woolf AD, Pfleger B: Burden of major musculoskeletal conditions. Bull World Health Organ 2003, 81(9):646-656.

40. Visentini PJ, Khan KM, Cook JL, Kiss ZS, Harcourt PR, Wark JD: The VISA score: an index of severity of symptoms in patients with jumper's knee (patellar tendinosis). Victorian Institute of Sport Tendon Study Group. J Sci Med Sport 1998, 1(1):22-28.

41. Bellamy N, Buchanan WW, Goldsmith CH, Campbell J, Stitt LW: Validation study of WOMAC: a health status instrument for measuring clinically important patient relevant outcomes to antirheumatic drug therapy in patients with osteoarthritis of the hip or knee. J Rheumatol 1988, 15(12):1833-1840.

42. Casey VA, Dwyer JT, Berkey CS, Coleman KA, Gardner J, Valadian I: Long-term memory of body weight and past weight satisfaction: a longitudinal follow-up study. Am J Clin Nutr 1991, 53(6):1493-1498.

43. Must A, Willett WC, Dietz WH: Remote recall of childhood height, weight, and body build by elderly subjects. Am J Epidemiol 1993, 138(1):56-64.

44. Perry GS, Byers TE, Mokdad AH, Serdula MK, Williamson DF: The validity of self-reports of past body weights by U.S. adults. Epidemiology 1995, 6(1):61-66.

doi:10.1186/1471-2474-15-266

Cite this article as: Fairley et al: Association between obesity and magnetic resonance imaging defined patellar tendinopathy in community-based adults: a cross-sectional study. BMC Musculoskeletal Disorders 2014 15:266.

\section{Submit your next manuscript to BioMed Central and take full advantage of:}

- Convenient online submission

- Thorough peer review

- No space constraints or color figure charges

- Immediate publication on acceptance

- Inclusion in PubMed, CAS, Scopus and Google Scholar

- Research which is freely available for redistribution 